\title{
Bioactivation of Aromatic Amines by Human CYP2W1, An Orphan Cytochrome P450 Enzyme
}

\author{
Chang-Yong Eun', Songhee Han', Young-Ran Lim', Hyoung-Goo Park', Jung-Soo Han', \\ Kyoung Sang Cho', Young-Jin Chun ${ }^{2}$ and Donghak Kim ${ }^{1}$ \\ 'Department of Biological Sciences, Konkuk University, Seoul 143-701 \\ ${ }^{2}$ College of Pharmacy, Chung-Ang University, Seoul 156-756, Korea
}

(Received July 29, 2010; Revised August 2, 2010; Accepted August 14, 2010)

\begin{abstract}
The human genome contains approximately 13 orphan cytochrome P450 (P450, CYP) genes, of which the apparent function or substrate has not been identified. However, they seem to possess their own biological relevance in some tissues or developmental stages. Here, we characterized the heterologously expressed CYP2W1, an orphan P450 enzyme. The recombinant CYP2W1 protein containing a $6 \times($ His)-tag at Nterminus has been expressed in Escherichia coli and purified. Expression level of CYP2W1 holoenzyme was around $500 \mathrm{nmol}$ P450 holoenzyme per liter culture medium. The reduced CO difference spectrum of CYP2W1 showed a maximum absorption at $449 \mathrm{~nm}$. CYP2W1 indicated the significant induction to bioactivate Trp-P-1, MeIQ, and IQ in E. coli DJ701 tester strain. However, the bioactivation of B[ $\alpha] \mathrm{P}$, and NNK by CYP2W1 was relatively low. The model structure of CYP2W1 suggested the characteristic P450 folds with the lengths and orientations of the individual secondary elements. The F-G loop is situated on the distal side of heme to accommodate the flexibility of active site of CYP2W1. These studies can provide useful information for the finding of its biological roles and structure-function relationships of an orphan CYP2W1 enzyme.
\end{abstract}

Key words: CYP2W1, Aromatic amines

\section{INTRODUCTION}

Cytochrome P450 (P450 or CYP) enzymes are found throughout all forms of life from bacteria to human (Guengerich, 1993). They are an enzyme superfamily of hemoproteins to catalyze the mixed-function oxidations (Guengerich, 2008). The human genomic analysis suggested that it has approximately 57 human P450 enzymes (Nelson et al., 2004). They are involved in the metabolism of endogenous or xenobiotic chemicals including various drugs. However, 13 of the human P450 genes can be considered "orphan" because presently their functions are unknown in terms of the oxidation of endogenous or xenobiotic substrates (Guengerich et al., 2005). The current list

Correspondence to: Donghak Kim, Department of Biological Sciences, Konkuk University, Seoul 143-701, Korea

E-mail: donghak@konkuk.ac.kr

Abbreviations: Trp-P-1, 3-amino-1,4-dimethyl-5H-pyrido[4,3,-b]indole; MelQ, 2-amino-3,5-dimethylimidazo[4,5-f]quinoline; IQ, 2amino-3-methylimidazo[4,5- $f$ ]quinoline; $B[a] P$, benzo[a]pyrene; NNK, 4-(methylnitrosamino)-1-(3-pyridyl)-1-butanone includes P450s 2A7, 2S1, 2U1, 2W1, 3A43, 4A22, 4F11, 4F22, 4V2, 4X1, 4Z1, 20A1, and 27C1 (Guengerich et al., 2005). Limited information is available for mRNA expression of these orphan P450s. The "deorphanization" of these enzymes is considered quite important for the use of pharmacological and clinical therapeutics (Guengerich et al., 2010b). Recently, several strategies for elucidating functions or substrates of the orphan P450s were proposed including metabolomic and isotopic labeling approaches (Guengerich et al., 2010a; Tang et al., 2009).

P450 2W1 is one of human orphan enzyme of which information has been relatively little known. mRNA expression of P450 2W1 were found in several adult tissues including spleen, testis, lung, pancreas, placenta, ovary, thymus, prostate, colon, and small intestine (Choudhary et al., 2005). Previous report showed that the heterologously expressed recombinant CYP2W1 exhibited broad catalytic activity towards several chemicals including benzphetamine, and arachidonic acid ( $\mathrm{Wu}$ et al., 2006). Their results indicated that CYP2W1 catalyzed bioactivation of several polycyclic hydrocarbons in bacterial umu system. Generally, dihydrodiols (benzo[ $\alpha]$ pyrene 7,8-diol) are more positive 
than the parent hydrocarbons because of absence of epoxide hydrolase in assay system. Trp-P-1, MeIQ, 2-aminofluorene, 2-aminoanthracene and benzo[ $\alpha]$ pyrene also showed limited activation in the test (Wu et al., 2006). The activation of a broad range of procarcinogens is of potential implication for the preferential expression of CYP2W1 in some tumors (Gomez et al., 2007; Karlgren et al., 2006).

Aromatic amines are of particular interest because of demonstrated carcinogenicity in animals and humans and the broad exposure to many of these compounds (Kim and Guengerich, 2005). P450 enzymes play a prominent role in the activation of these, usually involving critical $N$-hydroxylation. The resulting $N$-hydroxylamine products can be further activated to produce highly reactive ester derivatives that bind covalently to DNA via acetylation reaction by $N$-acetyltransferase (NAT). Josephy group developed the E. coli genotoxicity tester strains (DJ3109pNM12, and DJ701) carrying a (-GC) copy of lacZ gene can regain functional lacZ activity following induction of frameshifts by aromatic amines (Josephy, 2000; Zhou et al., 2004). This system allows that detection of mutagenic activation by recombinant P450 enzymes without a need for rat liver fractions.

In this investigation, we have characterized the purified CYP2W1 enzyme and examined its bioactivation activity of aromatic amines using E. coli DJ701 strain. These molecular analysis of CYP2W1 could provide the clue for the functional role of an orphan P450 enzyme.

\section{MATERIALS AND METHODS}

Chemicals. Sodium dithionite, NADP + , NNK, and $\mathrm{B}[\alpha] \mathrm{P}$ were purchased from Sigma (St. Louis, MO) or Aldrich Chemical Co. (Milwaukee, WI). IPTG was purchased from Anatrace (Maumee, OH). MeIQ, IQ, and Trp-P-1 were purchased from Toronto Research Chemicals (Toronto, Ontario, Canada). Other chemicals were of the highest grade commercially available.

Expression and purification of CYP2W1 enzyme. Expression and purification of the CYP2W1 enzyme were carried out as previously described with some modifications (Kim et al., 2005). Briefly, the E. coli strains transformed with $\mathrm{pCW}(2 \mathrm{~W} 1 / \mathrm{NPR} / \mathrm{His})$ vectors (Wu et al., 2006) were inoculated into TB medium containing $100 \mu \mathrm{g} / \mathrm{m} l$ ampicillin and $1.0 \mathrm{mM}$ IPTG. The expression cultures were grown at $37^{\circ} \mathrm{C}$ for $3 \mathrm{~h}$ and then at $28^{\circ} \mathrm{C}$ with shaking at $200 \mathrm{rpm}$ for $24 \mathrm{~h}$ in 1 liter Fernbach flasks. Bacterial inner membrane fractions containing CYP2W1 were isolated and prepared from 1 liter TB (with ampicillin, $100 \mu \mathrm{g} / \mathrm{m} l$ ) expression cultures of $E$. coli DH5 $\alpha$. Purification of CYP2W1 enzyme using a $\mathrm{Ni}^{2+}$-nitrilotriacetate column (Qiagen, Valencia, CA) was as previously described (Kim et al., 2005; Yun et al., 2000). Eluted fraction containing P450 dialyzed at $4^{\circ} \mathrm{C}$ against $100 \mathrm{mM}$ potassium phosphate buffer $(\mathrm{pH} 7.4)$ containing 20\% glycerol, $0.1 \mathrm{mM}$ EDTA.

Spectroscopic characterization of CYP2W1. P450 concentration was determined by the spectral method of Omura and Sato (Omura and Sato, 1964). Purified CYP2W1 was diluted with $100 \mathrm{mM}$ potassium phosphate buffer $(\mathrm{pH}$ 7.4). After adding small pieces of sodium dithionite crystals, a reference spectrum was recorded from 400 to $500 \mathrm{~nm}$ using a CARY Varian spectrophotometer (Palo Alto, CA). The solution was then saturated with carbon monoxide for $60 \mathrm{~s}$ and the spectrum was measured again. P450 concentration was calculated from the difference in absorbance between $450 \mathrm{~nm}$ and $490 \mathrm{~nm}$ with an extinction coefficient of $91 \mathrm{mM}^{-1} \mathrm{~cm}^{-1}$.<smiles>Cn1c(N)nc2c3cccnc3ccc21</smiles>

IQ<smiles>Cc1cc2ncccc2c2nc(N)n(C)c12</smiles>

MeIQ<smiles>Cc1c(N)nc(C)c2c1[nH]c1ccccc12</smiles>

TrpP-1<smiles>CN(CCCC(=O)c1cccnc1)N=O</smiles>

NNK<smiles>c1ccc2c(c1)cc1ccc3cccc4ccc2c1c34</smiles>

$\mathbf{B}[\boldsymbol{\alpha}] \mathbf{P}$

Fig. 1. Chemical structures of aromatic amines. 
Mutagenicity assays. A bicistronic $\mathrm{pCW}(2 \mathrm{~W} 1 / \mathrm{NPR} /$ His) vector DNA was transformed into E. coli DJ701 (Zhou et al., 2004) by a heat-shock method. The transformed strains were inoculated into $50 \mathrm{~m} l$ of TB expression medium and grown at $30^{\circ} \mathrm{C}$ for $24 \mathrm{~h}$. An aliquot of $500 \mu \mathrm{l}$ of each individual culture was applied onto minimal lactose agar medium in 36-square Integrid ${ }^{\mathrm{TM}}$ plates (Fisher Scientific, Pittsburgh, PA) using the $5 \mathrm{ml}$ top agar, which included aromatic amines. After $40 \mathrm{~h}$ of incubation at $37^{\circ} \mathrm{C}$, the number of revertant colonies was counted (Fig. 3).

Molecular modeling of CYP2W1. A molecular model of CYP2W1 was constructed using the human CYP2D6 crystal structure (pdb entry: $2 \mathrm{f} 9 \mathrm{q}$ ) as a template. The coordinates of the CYP2W1 model were obtained using software Swiss-Model from the Swiss Institute of Bioinformatics (http://swissmodel.expasy.org) (Arnold et al., 2006). The CYP2W1 model and the location of mutation were built using PyMOL software (DeLano Scientific, South San Francisco, CA).

\section{RESULTS AND DISCUSSION}

Expression and purification of recombinant CYP2W1. Recombinant CYP2W1 expression level spectrally determined in the whole cell culture was around $500 \mathrm{nmol}$ P450 holoenzyme per liter culture medium (Fig. 2A). It was simi- lar to the previously reported $350 \mathrm{nmol}$ per liter culture (Wu et al., 2006). After ultracentrifugation separation, the membrane fraction containing most of $\mathrm{P} 450$ protein was collected (Fig. 2B). An anionic surfactant, CHAPS, successfully solubilized the $\mathrm{P} 450$ protein in the membrane fraction. Purified CYP2W1 protein from Ni-column gave a single band on SDS-PAGE at $52 \mathrm{kDa}$ as expected with open reading frame (Fig. 2D).

Spectral characterization of purified CYP2W1. The reduced $\mathrm{CO}$ difference spectrum of CYP2W1 showed a maximum absorption at $449 \mathrm{~nm}$ (Fig. 2C). Examination of the absolute spectra showed that the ferric form of protein was almost low spin state (data not shown) as previously reported (Wu et al., 2006). Binding analysis of purified CYP2W1 with imidazole, and fluconazole showed the Type II binding spectra but, not spectral change was observed with itraconazole (data not shown). This result suggested that CYP2W1 has a typical water ligand binding in ferric form but the architecture in the active site for ligand binding is different from other P450 enzymes.

Mutagenicity activations. In order to evaluate the bioactivation of aromatic amines by CYP2W1, a $\beta$-galactosidase-based $E$. coli lacZ reversion mutagenicity test was used (Zhou et al., 2004). CYP2W1, NADPH-P450 reductase, and $\mathrm{N}$-acetyltransferase were expressed using a $\mathrm{pCW}$
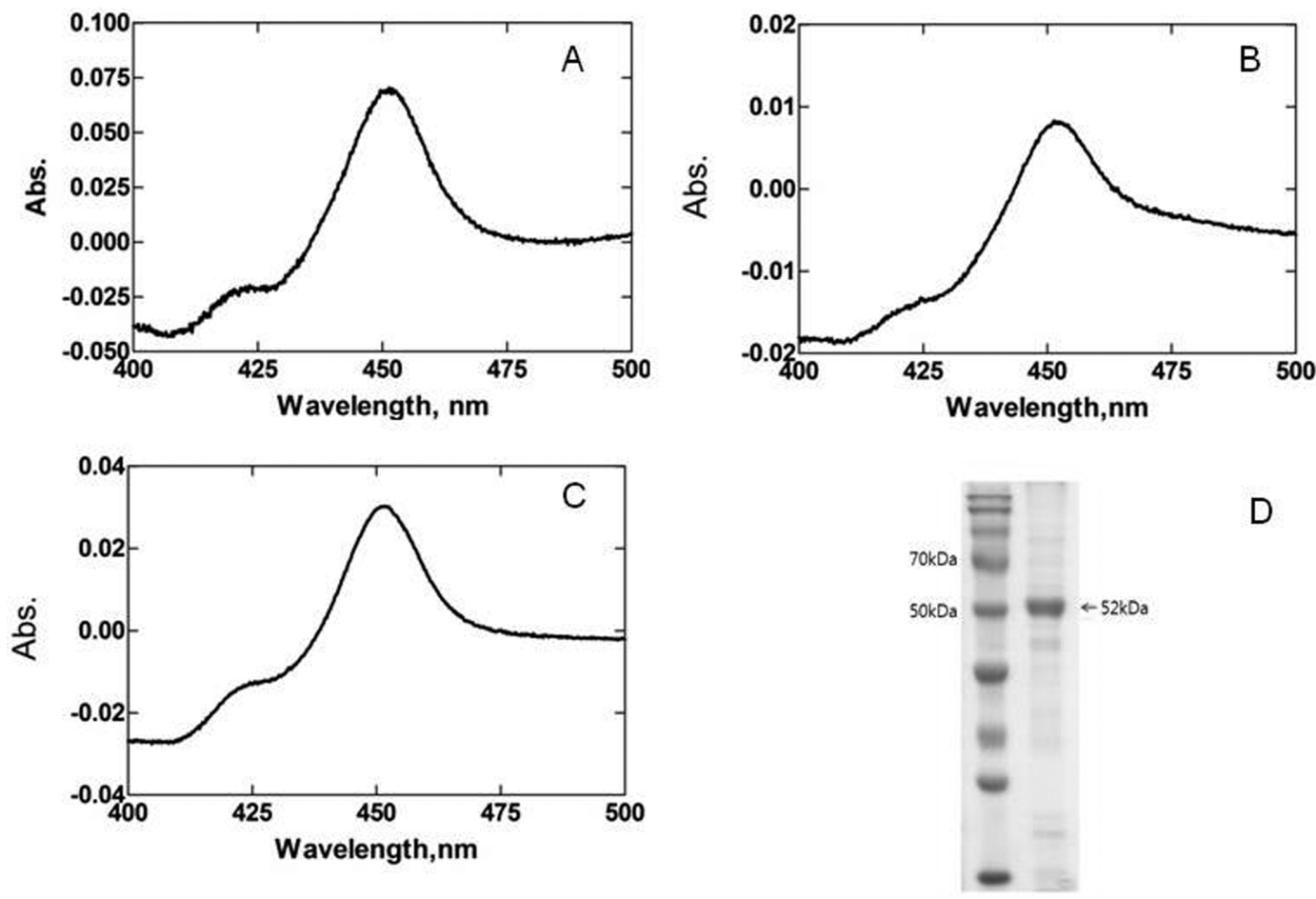

Fig. 2. Expression and purification of recombinant CYP2W1. (A) CO-binding spectrum of $2 \mathrm{~W} 1$ in E. coli whole cells. (B) CO-binding spectrum of 2W1 in membrane fractions. (C) CO-binding spectrum of purified 2W1. (D) an SDS-PAGE gel of the purified CYP2W1 protein. 


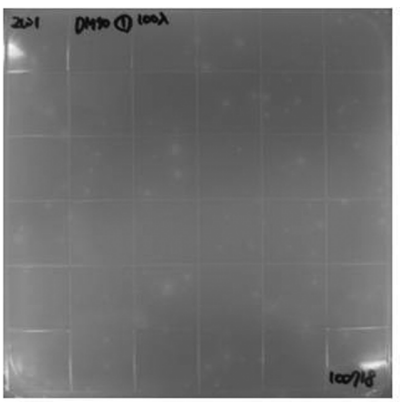

DMSO

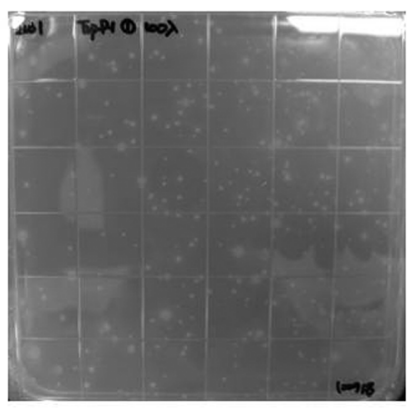

Trp-P-1
Fig. 3. Comparison of revertants bioactivated by Trp-P-1on minimal lactose media.

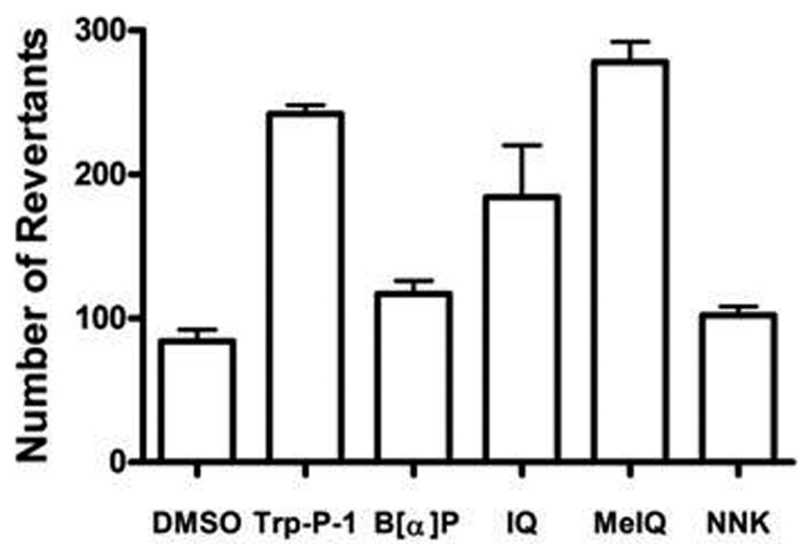

Fig. 4. Activation of aromatic amines by recombinant CYP2W1 in E. coli DJ701 genotoxicity system.

(2W1/NPR/His) plasmid and a pNM12 plasmid transformed E. coli DJ701 strain. Metabolic activation of aromatic amines by CYP2W1 induced (-2) frameshift mutations in the E. coli lac $\mathrm{Z}^{-}$strain and results in survival on lactose minimal medium. CYP2W1 showed the significant induction to activate Trp-P-1, MeIQ, and IQ (Fig. 4). However, the bioactivation of $\mathrm{B}[\alpha] \mathrm{P}$, and NNK by CYP2W1 was relatively low in E. coli DJ701 tester strain (Fig. 4). Previous study by Wu et al. also reported the Trp-P-1, and MeIQ were activated by CYP2W1 as well as several benzo[ $\alpha]$ pyrene diols (Wu et al., 2006). The results suggested that the expression of CYP2W1 in tumor specific tissues may be involved in the accumulation of mutation derived by the aromatic hydrocarbon compounds. They postulated that $\mathrm{B}[\alpha] \mathrm{P}$ diols was more active than parent $\mathrm{B}[\alpha] \mathrm{P}$ because the ити bacterial mutagenicity test strain was lack of epoxide hydrolase. E. coli DJ701 tester strain used in this study may have the same limitation therefore the low bioactivation of $\mathrm{B}[\alpha] \mathrm{P}$ was observed.

Homology modeling. The crystal structures of CYP2D6 in complex with a substrate were taken from the protein

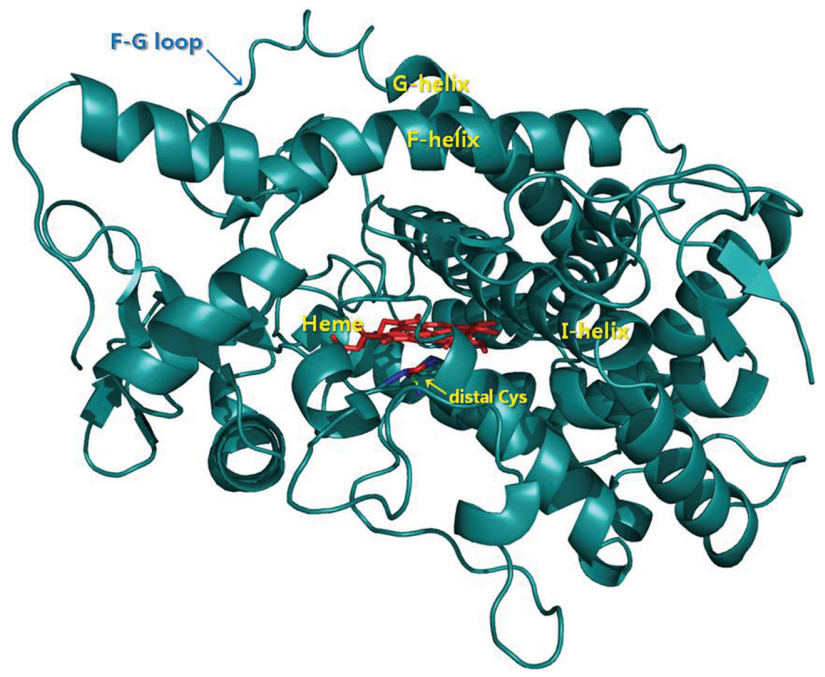

Fig. 5. Ribbon diagram of $C Y P 2 W 1$ model using the X-ray crystal structure of human CYP2D6 (PDB 2F9Q).

data bank (PDB, entry code 2F9Q) (Rowland et al., 2006) and used as the template for building the initial 3D model of human CYP2W1. The analyzed sequence identity was $39.9 \%$ between CYP2W1 and CYP2D6. The model structure of CYP2W1 shows the characteristic P450 fold with the lengths and orientations of the individual secondary elements (Fig. 5). The F-G loop is sutured on the distal side of heme and therefore it may accommodate the flexibility of active site of CYP2W1 (Fig. 5) as seen in the structure of CYP2D6 (Rowland et al., 2006). This model study indicated that CYP2W1 may bind various sizes of substrates in the enzyme. Previous model study using CYP2C5 also suggested $2 \mathrm{~W} 1$ possesses the regioselectivity toward benzphetamine, and indole with specific key residues including several Phe residues around heme ( $\mathrm{Li}$ et al., 2009). These residues were also observed in the active site of our CYP2W1 model (Fig. 1).

\section{CONCLUSIONS}

The "orphan" CYP2W1 was heterologously expressed and the holoenzyme containing heme was purified. Bioactivation of aromatic amines was analyzed using E. coli DJ701 strain. The model structure of CYP2W1 indicated the characteristic P450 folds with the lengths and orientations of the individual secondary elements. These studies can provide useful information for the finding of its biological roles and structure-function relationships of an orphan P450 enzyme.

\section{ACKNOWLEDGMENTS}

This work was supported by a Korea Research Foundation Grant funded by the Korean Government (MOEHRD, 
Basic Research Promotion Fund)(KRF-2008-331-E00030). The authors thank Prof. Zhong-Liu Wu for the initial CYP2W1 plasmid and Prof. David Josephy for E. coli strain DJ701.

\section{REFERENCES}

Arnold, K., Bordoli, L., Kopp, J. and Schwede, T. (2006). The SWISS-MODEL workspace: a web-based environment for protein structure homology modelling. Bioinformatics, 22, 195201.

Choudhary, D., Jansson, I., Stoilov, I., Sarfarazi, M. and Schenkman, J.B. (2005). Expression patterns of mouse and human CYP orthologs (families 1-4) during development and in different adult tissues. Arch. Biochem. Biophys., 436, 50-61.

Gomez, A., Karlgren, M., Edler, D., Bernal, M.L., Mkrtchian, S. and Ingelman-Sundberg, M. (2007). Expression of CYP2W1 in colon tumors: regulation by gene methylation. Pharmacogenomics, 8, 1315-1325.

Guengerich, F.P. (1993). Cytochrome P450 enzymes. American Scientist, 81, 440-447.

Guengerich, F.P. (2008). Cytochrome p450 and chemical toxicology. Chem. Res. Toxicol., 21, 70-83.

Guengerich, F.P., Tang, Z., Cheng, Q. and Salamanca-Pinzon, S.G. (2010a). Approaches to deorphanization of human and microbial cytochrome P450 enzymes. Biochim. Biophys. Acta., (in press).

Guengerich, F.P., Tang, Z., Salamanca-Pinzon, S.G. and Cheng, Q. (2010b). Characterizing proteins of unknown function: orphan cytochrome p450 enzymes as a paradigm. Mol. Interv., 10, 153163.

Guengerich, F.P., Wu, Z.L. and Bartleson, C.J. (2005). Function of human cytochrome P450s: characterization of the orphans. Biochem. Biophys. Res. Commun., 338, 465-469.

Josephy, P.D. (2000). The Escherichia coli lacZ reversion mutagenicity assay. Mutat. Res., 455, 71-80.

Karlgren, M., Gomez, A., Stark, K., Svard, J., Rodriguez-Antona, C., Oliw, E., Bernal, M.L., Ramon y Cajal, S., Johansson, I. and Ingelman-Sundberg, M. (2006). Tumor-specific expression of the novel cytochrome P450 enzyme, CYP2W1. Biochem. Bio- phys. Res. Commun., 341, 451-458.

Kim, D. and Guengerich, F.P. (2005). Cytochrome P450 activation of arylamines and heterocyclic amines. Annu. Rev. Pharmacol. Toxicol., 45, 27-49.

Kim, D., Wu, Z.L. and Guengerich, F.P. (2005). Analysis of coumarin 7-hydroxylation activity of cytochrome P450 2A6 using random mutagenesis. J. Biol. Chem., 280, 40319-40327.

Li, W., Tang, Y., Hoshino, T. and Neya, S. (2009). Molecular modeling of human cytochrome P450 2W1 and its interactions with substrates. J. Mol. Graph. Model, 28, 170-176.

Nelson, D.R., Zeldin, D.C., Hoffman, S.M., Maltais, L.J., Wain, H.M. and Nebert, D.W. (2004). Comparison of cytochrome P450 (CYP) genes from the mouse and human genomes, including nomenclature recommendations for genes, pseudogenes and alternative-splice variants. Pharmacogenetics, 14, 118 .

Omura, T. and Sato, R. (1964). The Carbon Monoxide-Binding Pigment of Liver Microsomes. I. Evidence for Its Hemoprotein Nature. J. Biol. Chem., 239, 2370-2378.

Rowland, P., Blaney, F.E., Smyth, M.G., Jones, J.J., Leydon, V.R., Oxbrow, A.K., Lewis, C.J., Tennant, M.G., Modi, S., Eggleston, D.S., Chenery, R.J. and Bridges, A.M. (2006). Crystal structure of human cytochrome P450 2D6. J. Biol. Chem., 281, 7614-7622.

Tang, Z., Martin, M.V. and Guengerich, F.P. (2009). Elucidation of functions of human cytochrome P450 enzymes: identification of endogenous substrates in tissue extracts using metabolomic and isotopic labeling approaches. Anal. Chem., 81, 3071-3078.

Wu, Z.L., Sohl, C.D., Shimada, T. and Guengerich, F.P. (2006). Recombinant enzymes overexpressed in bacteria show broad catalytic specificity of human cytochrome P450 2W1 and limited activity of human cytochrome P450 2S1. Mol. Pharmacol., 69, 2007-2014.

Yun, C.-H., Miller, G.P. and Guengerich, F.P. (2000). Rate-determining steps in phenacetin oxidations by human cytochrome P450 1A2 and selected mutants. Biochemistry, 39, 1131911329.

Zhou, H., Josephy, P.D., Kim, D. and Guengerich, F.P. (2004). Functional characterization of four allelic variants of human cytochrome P450 1A2. Arch. Biochem. Biophys., 422, 23-30. 